\title{
Rosa la cigarrera de Madrid (1872) de Faustina Sáez de Melgar como modelo literario de La Tribuna (1883) de Emilia Pardo Bazán
}

\author{
Cristina Enríquez de Salamanca
}

Emilia Pardo Bazán publica La Tribuna en $1883^{\mathrm{I}}$, novela que la mayoría de la crítica considera integrante del ciclo naturalista de esta autora ${ }^{2}$. La Tribuna narra la historia de Amparo, cigarrera en la Fábrica de Tabacos de Marineda (La Coruña), que participa en el movimiento federalista, es seducida por un hombre que la abandonará y acaba dando a luz un hijo en el momento en que se proclama la Primera República (1873).

Las declaraciones de la autora en torno al proceso creativo de esta obra, contenidas en el "Prólogo" de la novela, en los Apuntes autobiográficos y en las declaraciones a la revista Madrid Cómico en $1898^{3}$, apuntan en dos direcciones. La primera destinada a insertar La Tribuna en la estela de la tradición literaria masculina representada por obras y escritores tales como $L a$ desheredada de Benito Pérez Galdós, L'Assommoir de Emile Zola y Germinie Lacerteux de Edmond y Jules Goncourt, así como el escritor José María Pereda. La segunda, dirigida a poner de manifiesto que La Tribuna nace del propósito de "estudiar el desarrollo de una creencia política en un cerebro de hembra" 4 por medio de técnicas propias del naturalismo, la observación directa, el análisis y la documentación.

\footnotetext{
${ }^{\text {I }}$ Aunque en la primera edición de La Tribuna no consta fecha de edición y el prólogo está fechado en 1882, sin duda alguna la novela apareció en 1883 (González Herrán 1983: 259-287). Agradezco sinceramente al profesor González Herrán su interés en la publicación de este trabajo, inicialmente presentado como ponencia en la primavera de 1989 en la Sixth Annual Wichita State University Conference on Foreign Literatures.

${ }^{2}$ Pattison (1971); Varela Jácome (1973); Osborne (1964); Clémessy (1981). A propósito del discutido naturalismo de La Tribuna Round (1983: 325-343); Clémessy (1988: 485 496); González Herrán (1988: 497-512); Sobejano (1988: 583-615). Para una revisión reciente de los estudios críticos, José Manuel Gónzález Herrán (2007: 29-39).

3 "Prólogo de la autora a la primera edición," (Pardo Bazán 1982: 57-59), en adelante cito de esta edición de Varela Jácome; los "Apuntes autobiográficos", publicados como prólogo de la primera edición de Los Pazos de Ulloa (Pardo Bazán 1886: 5-92) se recogen en el tomo III de las Obras Completas (1973: 698-732). Madrid Cómico (1898: 318); rep. Pattison (1971: 120-121).

4 “Apuntes” (Pardo Bazán 1973: 725).
}

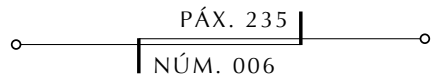


Las declaraciones de Pardo Bazán no sugieren otra dirección que la indicada en cuanto a la filiación de esta novela o de su protagonista. González Herrán, que ya en 1988 apuntaba la influencia en La Tribuna de ciertos elementos de folletín romántico, consideró la posibilidad de que la autora hubiera tomado como modelo para la protagonista una popular cigarrera santanderina conocida como "La Republicana" 5 .

Mi propuesta relaciona La Tribuna con la novela de Faustina Sáez de Melgar, Rosa la cigarrera de Madrid (Barcelona, 1872) ${ }^{6}$. La figura de Faustina Sáez de Melgar (1834-1895) ha sido recuperada para la historia literaria junto a otras autoras cuyas obras ocupan la segunda mitad del siglo XIX, muy especialmente María del Pilar Sinués de Marco (1835-1893) y Ángela Grassi de Cuenca (1823-1883)7. El redescubrimiento de la tradición literaria femenina de la segunda mitad del siglo XIX se ha realizado principalmente bajo el signo de los estudios culturales y de género y de la historia de las ideas estéticas y los géneros literarios en la cultura del XIX. Así, se ha enmarcado a Sáez de Melgar como uno de los paradigmas de las escritoras domésticas o escritoras isabelinas, según la perspectiva adoptada. La primera denominación responde a los estudios de género, que han atribuido a la escritura doméstica un papel relevante en la construcción del modelo de feminidad propio del liberalismo, simbolizado en el icono del ángel del hogar ${ }^{8}$. Escritoras isabelinas denomina Íñigo Sánchez Llama a aquellas que, nuevamente representadas por Sáez de Melgar, Sinués y Grassi, publican durante el reinado de Isabel II (1843-1868) adaptando su escritura a un criterio estético dominante en este periodo, que otorga "sublimidad artística a la transmisión de doctrina cristiana y contenidos moralizantes9." La estética isabelina identifica "lo bueno" con

5 González Herrán (1975: 6-7); (1988: 497-512); este último trabajo había sido ponencia en un congreso celebrado en noviembre de 1987.

${ }^{6}$ Rosa la cigarrera de Madrid. Gran novela original de la señora Doña Faustina Sáez de Melgar (1872): Barcelona: Biblioteca Hispano-Americana. Hay una edición del primer tomo de 1878 en la misma editorial. Todas las referencias del texto son a la edición de 1872 .

${ }^{7}$ Enríquez de Salamanca (1989: 81-107). Este artículo representó mi primera aproximación a las escritoras del XIX. Para un análisis de las obras de Sáez de Melgar mi artículo, “Faustina Sáez de Melgar”, en Linda Levine (1993: 460-472). Para las escritoras del XIX es imprescindible Simón Palmer (1991).

8 Aldaraca (1992); Jagoe (1994); Jagoe, Blanco y Enríquez de Salamanca (1998); Charnon Deutsh y Labanyi (1995); Charnon Deutsh (1994); Blanco (2001).

9 Sánchez Llama (2000: 14). La formación del canon literario y consiguiente exclusión de las escritoras se analiza en Jagoe (1993: 427-435) y (1993: 227-248); Blanco (1993: 463-470). 
"lo bello" y "lo malo" con lo defectuoso, es decir, la representación de los valores morales con la belleza y la falta de valor estético con la textualización del mal, todo esto entendido en la visión del mundo del catolicismo en la sociedad del XIX.

Faustina Sáez de Melgar nace en Villamanrique de Tajo (Madrid) en 1834 y muere en Madrid en 1895. Perteneciente a una familia de propietarios rurales, contrae matrimonio en 1855 con el periodista Valentín Melgar y desarrolla una extensa carrera literaria ${ }^{\mathrm{IO}}$. En su producción se incluyen obras poéticas, didácticas, literatura infantil, traducciones y una veintena de novelas publicadas en la Biblioteca de Señoras y en las páginas de diarios y revistas como La Iberia, El Correo de la Moda y La Violeta (Madrid 1862-1866), de la cual fue directora y propietaria. En las obras de Faustina Sáez de Melgar se observa una combinación de elementos: los propios de la novela del folletín, desarrollados en episodios de la historia contemporánea, con frecuencia las guerras carlistas; una técnica estilística que se aproxima a un realismo esquemático, una preocupación esencial por el mundo de la mujer y una ideología fluctuante, cuyas oscilaciones bien pueden deberse a los vaivenes políticos propios de la época.

Rosa la cigarrera de Madrid se publica cuando la carrera literaria de su autora está plenamente consolidada y su talante es un resultado de la revolución de 1868. Escrita con un propósito didáctico -"la instrucción de esa clase popular á la que está más plenamente dedicada"- (1872: I, 72), adopta una posición de clara defensa de las clases populares y de la ideología liberal y se define contra una aristocracia representada en los términos del absolutismo del Antiguo Régimen y de las propuestas políticas del carlismo. La acción de esta extensa novela en dos tomos transcurre entre la primera guerra carlista en los años treinta del siglo pasado y la revolución liberal de 1854 que, encabezada por los generales O'Donnell y Espartero, y con el apoyo del pueblo de Madrid, acabó con once años de gobierno conservador y condujo a la expatriación de la Reina Madre María Cristina de Habsburgo. La acción transcurre entre las zonas rurales de Huesca, en el primer tomo, y la ciudad de Madrid, en el segundo tomo. La trama sigue las historias

\footnotetext{
Io María del Carmen Simón Palmer cita como primera publicación, la de la poesía "La paloma torcaz," en El Álbum de señoritas, el 28 de febrero de 1852, p. 35 y afirma, sin dar la cita exacta, que éste poema había sido publicado hacia 1851 en El Correo de la Moda. La obra de Simón Palmer contiene una extensa bibliografía de Faustina Sáez de Melgar (1991: 607-618). Isabel Abradelo de Usera (1998) dedicó su tesis doctoral a esta escritora, Faustina Sáez de Melgar. También en Abradelo (2000: 811-832).
} 
entrelazadas de dos personajes principales: Rosa, una mujer del pueblo, y Tula, una aristócrata, quienes al modo de Fortunata y Jacinta comparten al mismo hombre, Jaime, como amante y marido respectivamente. La línea principal de la novela narra la vida de Rosa, desde su seducción inicial por Jaime cuando es una adolescente, su conversión en capitana de una tropa de facciosos a raíz del abandono de su amante, el traslado a Madrid donde trabaja como cigarrera en la fábrica de Tabacos de la calle Embajadores, su activa participación en los motines revolucionarios de Madrid en 1854 y el matrimonio final con un Jaime arrepentido.

Las características de la novela de Sáez de Melgar que permiten relacionarla con La Tribuna se refieren tanto al plano general de construcción de la obra como a varios elementos específicos: por ejemplo, las protagonistas como víctimas de una seducción amorosa y la descripción del grupo social al que pertenecen, las cigarreras. En el plano general encontramos tres puntos de coincidencia: la reconstrucción de acontecimientos de la historia española; el hecho de que tales acontecimientos jueguen un papel importante en la vida de los personajes, y el tratamiento del ambiente popular urbano.

Respecto al primer punto, ambas escritoras se documentaron en la prensa de la época. Sáez de Melgar cita como fuente para la descripción de la Revolución de 1854 "las páginas ilustradas que escribió D. Vicente Barrantes y se publicaron en "La Ilustración" (1872: II, 579) y Pardo Bazán se refiere en los Apuntes autobiográficos a la investigación en la prensa local de la época.

En segundo lugar, la participación de la protagonista en los acontecimientos políticos del periodo 1837-1854 determinan el desarrollo de Rosa la cigarrera, de la misma forma que la vida de Amparo gira en torno a la revolución de 1868. Así como el parto de Amparo coincide con la proclamación de la Primera República, la novela de Sáez de Melgar abunda en estas coincidencias, de las que daremos un ejemplo: Rosa, "Armada de un agudo puñal penetró en su cuarto; era el 29 de setiembre (sic) de 1840 y en aquel momento entraba en Madrid el general Espartero ..." (Sáez de Melgar 1872: II, 111).

Finalmente, el tratamiento del ambiente popular urbano se manifiesta en Rosa la cigarrera en la presencia de Madrid y de sus calles, La Montera, Duque de Alba, Fuencarral, Embajadores y Toledo; también en sus viviendas y personajes -artesanos, comerciantes, truhanes, empleadas- designados con motes característicos. Pardo Bazán por su parte, nos sitúa en La Coruña del ochocientos y a través de los barrios, calles y edificios de la ciudad, nos pone en contacto con sus habitantes. 
Los puntos concretos por los que la obra de Sáez de Melgar puede considerarse modelo de La Tribuna son: el carácter de las protagonistas, el hecho de que las dos son seducidas por un hombre de nivel social superior que las abandona; la presentación de una comunidad de cigarreras y en ella, de un motín; el análisis de la problemática obrera de la mujer y la participación de las mujeres en actividades políticas.

Rosa y Amparo aparecen en la ficción en el momento de su paso a la madurez sexual. Rosa tiene quince años y Amparo trece. Ambas son mujeres altas, fuertes, de carácter violento; Rosa con "mirada de leona" (Sáez de Melgar 1872: I, 12) y Amparo con "melena leonada" (Pardo Bazán 1982: 190, 258). Las dos aprenden a leer y están insatisfechas con las limitadas expectativas vitales de las clases populares. Las dos son seducidas por un hombre que desarrolla una complicada estrategia y las dos también ceden ante la promesa de matrimonio.

La descripción de la vida fabril y el motín de las cigarreras ocupan el capítulo I del primer tomo y los capítulos IX y X del segundo tomo de la obra de Sáez de Melgar. La fábrica de tabacos de la calle Embajadores de Madrid es el elemento físico descrito con más detalle de toda la novela, atendiendo a sus características arquitectónicas, material de construcción, longitud y fecha de construcción. Este detallismo, ausente en el resto de la novela, confiere una importancia simbólica al edificio, elemento que recogerá La Tribuna. Sáez de Melgar señala el gran número de trabajadoras, 4.000 (número que coincide con el de la fábrica de tabacos de Marineda) ${ }^{\mathrm{II}}$ y la existencia entre ellas de jerarquías, debido tanto a los puestos de trabajo como a los talleres a los que pertenecen $(I, 58)$; también señala la dureza de las condiciones de trabajo y las distintas clases de labores realizadas en la fábrica. Todos estos detalles indican un conocimiento real de la vida fabril, que Emilia Pardo Bazán amplía en La Tribuna. El motín de cigarreras se produce en ambas novelas por motivos económicos: la reducción del número de horas de trabajo y del precio de las labores en Rosa la cigarrera y el retraso en el pago de los haberes en La Tribuna. Rosa encabeza el motín, como Amparo, y en ambas novelas la rebelión acaba con la intervención de las tropas y a continuación las protagonistas sufrirán represalias.

II Sáez de Melgar (1872: I, 12); Pardo Bazán (1982: 190 y 258). 


\section{Tributa

La existencia de una problemática obrera, señaladamente repetida como una innovación de La Tribuna en la novelística española ${ }^{\mathrm{I2}}$, aparece ya en Rosa la cigarrera. Esta obra reivindica a las cigarreras como grupo social desprestigiado, representa la dureza de la vida de las clases populares en un afán de justicia social y ensalza el valor del trabajo mientras que denigra al estamento aristocrático, aunque la explicación de la desigualdad social como resultado de la explotación de la clase obrera está planteada más explícitamente en La Tribuna.

Por último, ambas novelas coinciden en la presentación de un violento colectivo femenino en sucesos revolucionarios. La lucha del pueblo de Madrid se contiene en los capítulos XCII a CII del segundo tomo de Rosa la cigarrera. Las mujeres del pueblo se enfrentan a la guardia del Palacio Real, forzando la entrada, destrozando el mobiliario y haciendo hogueras en las calles (Sáez de Melgar 1872: II, 550-51), en una explosión de violencia que Pardo Bazán modera, porque el federalismo de las cigarreras de Marineda existe solamente a nivel verbal, y su única expresión pública consiste en el recibimiento de los delegados federalistas de Cantabria y la asistencia a la firma del acuerdo unionista.

Las dos novelas relacionan la condición femenina con la esfera pública. La conversión de Rosa en capitana de bandidos se produce cuando, al ser abandonada por Jaime, rechaza su identidad femenina; las palabras de Rosa son inequívocas: "Ea esclamaba (sic) con alborozo; ya soy otra mujer; o mejor dicho, ya no soy otra mujer, soy hombre!" (Sáez de Melgar 1872: I, 308); Amparo, por su parte, abandona su actividad política cuando comienza su relación con Baltasar Sobrado. Sáez de Melgar sugiere una idea desarrollada más explícitamente por Pardo Bazán: la situación de la mujer no depende de la vigencia de uno u otro régimen político, ya que, el patriarcado es transversal. Para Rosa, como para Amparo, el enemigo no es la tiranía, sino el hombre y la doble moral sexual. La solución de los problemas de Rosa no viene del cambio en las ideas políticas de su seductor, sino de una decisión personal de éste; la revolución liberal trae libertad política a la sociedad, pero no justicia para Rosa, en cuando madre soltera y mujer deshonrada. Esta

I2 Han señalado y estudiado la temática obrera y la dimensión socio-política en $L a$ Tribuna Fuentes (1971: 90-94); Gullón (1976: 43-64); Henn (1984: 77-90); Miranda García (1987: 103-114); Sánchez Reboredo (1979: 567-580); Scanlon (1989: 289-299); Whitaker (1988: 71-80). 
idea aparece en la obra de Pardo Bazán, que manifiesta la injusticia de una sociedad que permite a los hombres abandonar impunemente a la mujer que han seducido y al hijo engendrado.

Encontramos, pues, una serie de elementos comunes entre Rosa la cigarrera y La Tribuna, obras que coinciden en su apreciar, desde tan distantes posiciones, pareja sensibilidad, por la situación de la mujer. Ciertos trabajos han señalado que Pardo Bazán, aunque ciertamente conoció a Sáez de Melgar y otras autoras, evitó cuidadosamente alinearse con las literatas, de la misma forma que se conoce el distanciamiento de las propias literatas respecto a Pardo Bazán, especialmente por la aproximación de ésta al naturalismo. Las relaciones entre ellas se pueden caracterizar por el silencio y la incomodidad, cuando no hostilidad, que son también fácilmente perceptibles en la historia literaria respecto a la novela sentimental y los folletines decimonónicos; también respecto a las propias literatas, tanto en los estudios de género, como en los culturales y en al ámbito académico, que desdeña las obras de las escritoras y ha tendido a descalificarlas globalmente ${ }^{\mathrm{I3}}$.

La problemática situación social de la mujer aparece en la escritura del XIX con tanta truculencia en la novela naturalista como en la realista. En los folletines del socialismo utópico de un Wenceslao Ayguals de Izco y en los folletines "sentimentales" de las literatas abundan los temas tabú: hijos ilegítimos, sexualidad, incesto y violencia; en las obras de las literatas como Faustina Sáez de Melgar y no digamos en Pilar Sinués se perciben subtextos feministas, rechazos del rol doméstico asignado a la mujer en la sociedad, relaciones matrimoniales cargadas de frustración. La intensidad con que se retrata el mundo femenino, en toda su complejidad, constituye una novedad en la historia literaria y aporta fuerza y fascinación a la escritura doméstica. No puede extrañar, por tanto, que Emilia Pardo Bazán conociera las novelas, folletines, poesía y manuales de las literatas y como en este trabajo propongo, en particular la escritura de Faustina Sáez de Melgar.

\footnotetext{
I3 A propósito de la relación entre Pardo Bazán y Sáez de Melgar, es seguro que se conocieron y mantuvieron correspondencia hacia 1881-1882, pues doña Emilia colaboró con dos artículos ("La cigarrera" y "La gallega") en el álbum costumbrista Las mujeres españolas, americanas y lusitanas pintadas por sí mismas... [s. a.], publicado bajo la dirección de doña Faustina. En este tema Bieder (1992: 1203-1212).
}

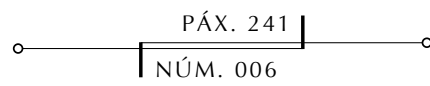




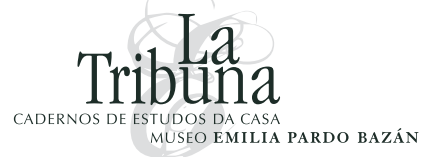

BIBLIOGRAFÍA

- Abradelo de Usera, Isabel (1998): "Faustina Sáez de Melgar (1834-1895)", en Literatura, sociedad y mujer en la segunda mitad del siglo XIX, Universidad Complutense de Madrid, Filología.

- Abradelo de Usera, Isabel (2000): “Una mujer escritora del siglo XIX: Faustina Sáez de Melgar (1834-1895)", Anuario jurídico y económico escurialense, 33, pp. 811-832.

- Aldaraca, Bridget (1992): El ángel del hogar: Galdós and the Ideology of Domesticity in Spain, Chapell Hill, Carolina del Norte, University of North Carolina Press.

- Bieder, Maryellen (1992): "Emilia Pardo Bazán y las literatas: las escritoras españolas del siglo XIX y su literatura", en Antonio Vilanova (ed.): Actas del $X$ Congreso de la Asociación Internacional de Hispanistas, Barcelona PPU, pp. 1203-1212.

- Blanco, Alda (1993): "But are they Any Good?", Revista de Estudios Hispánicos, 27, 1993, pp. 463-470.

- Blanco, Alda (2001): Escritoras virtuosas: narradoras de la domesticidad en la España isabelina, Granada, Universidad de Granada.

- Charnon Deutsch, Lou (1994): Narratives of Desire. Nineteenth-Century Spanish Fiction by Women, Pennsylvania, University Park, The Pennsylvania State University Press.

- Charnon Deutsch, Lou y Jo Labanyi (eds.) (1995): Culture and Gender in Nineteenth-Century Spain. Oxford, Clarendon Press.

- Clemessy, Nelly (1981): Emilia Pardo Bazán como novelista (De la teoría a la práctica), Madrid, Fundación Universitaria Española.

- Clemessy, Nelly (1988): "De La cuestión palpitante a La Tribuna: teoría y praxis de la novela de Emilia Pardo Bazán", en Y. Lissorgues (ed.): Realismo y Naturalismo en España en la segunda mitad del siglo XIX, Barcelona, Anthropos, pp. 485-496.

- Enríquez de Salamanca, Cristina (1989): “¿Quién era la escritora del siglo XIX?", Letras Peninsulares, pp. 81-107.

- Enríquez de Salamanca, Cristina (1993): "Faustina Sáez de Melgar", en Linda Levine et al. (eds.): Spanish Women Writers. A Bio-Bibliographical Source Book, Westport, Connecticut y Londres, Greenwood Press, pp. 460472.

- Fuentes, Víctor (1971): "La aparición del proletariado en la novelística. Sobre La Tribuna", Grial, 31, pp. 90-94.

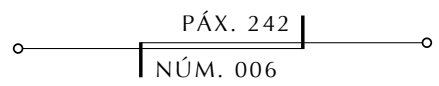


- González Herrán, José Manuel (1975): "La Tribuna, de Emilia Pardo Bazán y un posible modelo real de su protagonista", Ínsula 346, 1, 6-7.

- González Herrán, José Manuel (1983): “Emilia Pardo Bazán y José María de Pereda: algunas cartas inéditas", BBMP, LIX, pp. 259-287.

- González Herrán, José Manuel (1988): "La Tribuna, de Emilia Pardo Bazán, entre romanticismo y naturalismo", en Y. Lissorgues (ed.): Realismo y Naturalismo en España en la segunda mitad del siglo XIX, Barcelona, Anthropos, pp. 497-512.

- Gónzález Herrán, José Manuel (2007): “Lecturas críticas de La Tribuna, de Emilia Pardo Bazán 1883-2008", La Tribuna. Cadernos de Estudos da Casa Emilia Pardo Bazán, 5, pp. 29-39.

- Gullón, Germán (1976): “¿Es La Tribuna novela social?”, en El narrador en la novela del siglo XIX, Madrid, Taurus, pp. 43-64.

- Henn, David (1984): "Aspectos políticos de La Tribuna, de Emilia Pardo Bazán", en J. M. Ruiz Veintemilla (ed.): Estudios dedicados a James Leslie Brooks, Barcelona, Puvill Libros, pp. 77-90.

- Jagoe, Catherine (1993): "Disinheriting the Feminine: Galdós and the Rise of realist Novel in Spain", Revista de Estudios Hispánicos, 27, pp. 227-248.

- Jagoe, Catherine (1993): "Noncanonical Novels and the Question of Quality", Revista de Estudios Hispánicos, 27, pp. 427-435.

- Jagoe, Catherine (1994): Ambiguous Angels. Gender in the Novels of Galdós, Berkeley y Los Angeles, University of California Press.

- Jagoe, Catherine, Alda Blanco y Cristina Enríquez de Salamanca (1998): La mujer en los discursos de género, Barcelona, Icaria.

- Miranda García, Soledad (1985): "La cuestión social en la novela española: La Tribuna (1882), La Espuma (1891), El Intruso (1904)", en El reformismo social en España: La comisión de Reformas Sociales. Actas de los IV Coloquios de Historia [Madrid, 1985], Córdoba, Monte de Piedad y Caja de Ahorros de Córdoba, pp. 103-114.

- Osborne, Robert (1964): Emilia Pardo Bazán. Su vida y sus obras, México, Ediciones de Andrea.

- Pardo Bazán, Emilia (1973): Obras Completas, III, Madrid, Aguilar, pp. 698-732.

- Pardo Bazán, Emilia (1982): "Prólogo de la autora a la primera edición", Benito Varela Jácome (ed.): La Tribuna, 4ª edición, Madrid, Cátedra, pp. 57-59.

- Pardo Bazán, Emilia (1886): "Apuntes autobiográficos", Los Pazos de Ulloa, Barcelona, Cortezo, pp. 5-92. 
- Pattison, Walter T. (1971): Emilia Pardo Bazán, New York, Twayne Publishers, Inc.

- Round, Nicholas G. (1983): "Naturalismo e ideología en La Tribuna", Estudios ofrecidos a Emilio Alarcos Llorach, V, Oviedo, Universidad, pp. 325 343.

- Sáez de Melgar, Faustina (1852): "La paloma torcaz," El Álbum de señoritas, 28 de febrero, p. 35.

- Sáez de Melgar, Faustina (1872): Rosa la cigarrera de Madrid. Gran novela original de la señora Doña Faustina Sáez de Melgar, Barcelona, Biblioteca Hispano-Americana.

- Sánchez Llama, Iñigo (2000): Galería de escritoras isabelinas. La prensa periódica entre 1833 y 1895, Madrid, Ediciones Cátedra, Universitat de València, Instituto de la Mujer.

- Sánchez Reboredo, José (1979): “Emilia Pardo Bazán y la realidad obrera: notas sobre La Tribuna", Cuadernos Hispanoamericanos, 351, pp. 567-580.

- Scanlon, Geraldine M. (1988): "Ideología y experiencia femenina en La Tribuna de Emilia Pardo Bazán", en V. Maquieira D’Angelo, G. GómezFerrer Morant, M. Ortega López (eds.): Mujeres y hombres en la formación del pensamiento occidental (Actas de las VII Jornadas de Investigación Interdisciplinaria), Vol. II, Madrid, Instituto Universitario de Estudios de la Mujer-Universidad Autónoma de Madrid, pp. 289-299.

- Simón Palmer, María del Carmen (1991): Escritoras españolas del siglo XIX. Manual Bio-Bibliográfico, Madrid, Castalia, 607-618.

- Sobejano, Gonzalo (1988): "El lenguaje de la novela naturalista", en Y. Lissorgues (ed.): Realismo y Naturalismo en España en la segunda mitad del siglo XIX, Barcelona, Anthropos, 583-615.

- Varela Jácome, Benito (1973): Estructuras novelísticas de Emilia Pardo Bazán. La Coruña, Santiago de Compostela, C.S.I.C.

- Whitaker, Daniel S. (1988): "Power and Persuasión in Pardo Bazán’s La Tribuna", Hispanic Journal, 9, 2, pp. 71-80. 